\title{
Studying the role of CgMIP1 in the evolution of Candida glabrata during adaption to the human host
}

Sofia Siscar-Lewin', Sascha Brunke', Bernhard Hube ${ }^{1,2,3}$

1Bepartment of Microbial Pathogenicity Mechanisms, Hans-Knöll-Institute, Jena, Germany; 2 Friedrich-Schiller-University Jena, Germany

\section{Introduction}

Candida glabrata is the second most prevalent cause of candidemia, mainly due to its intrinsic and rapidly acquired resistance to azole antifungals. Phylogenetically, C. glabrata is more closely related to the baker's yeast Saccharomyces cerevisiae than to the most common Candida species, C. albicans. Recent evolutionary studies have shown that the gene CgMIP1 may have been under high selective pressure during the evolution of $C$. glabrata as a human pathogen. The ortholog MIP1 in S. cerevisiae encodes a mitochondrial DNA polymerase.

Defects in mitochondrial functions cause respiratory deficiency and lead to a petite phenotype in C. glabrata and S. cerevisiae. This is linked to increased resistance against azoles, such as fluconazole (FL), due to the overexpression of the transcriptional factor Pdr1. In addition, petite clinical isolates of C. glabrata have been reported, but data on their virulence is controversial. We study whether the rapidly evolved gene CgMIP1 is involved in the adaptation to the human host and pathogenicity of C. glabrata.

\section{Phenotypic characterization of mip1 $\Delta$ from C. glabrata and S. cerevisiae}

Loss of mitochondrial function

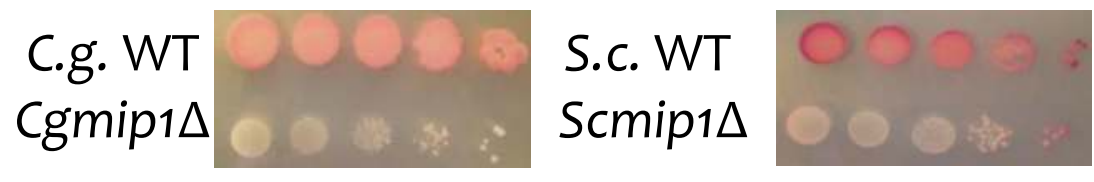

Increased resistance to ER stress and fluconazole

YPD Tunicamycin DTT

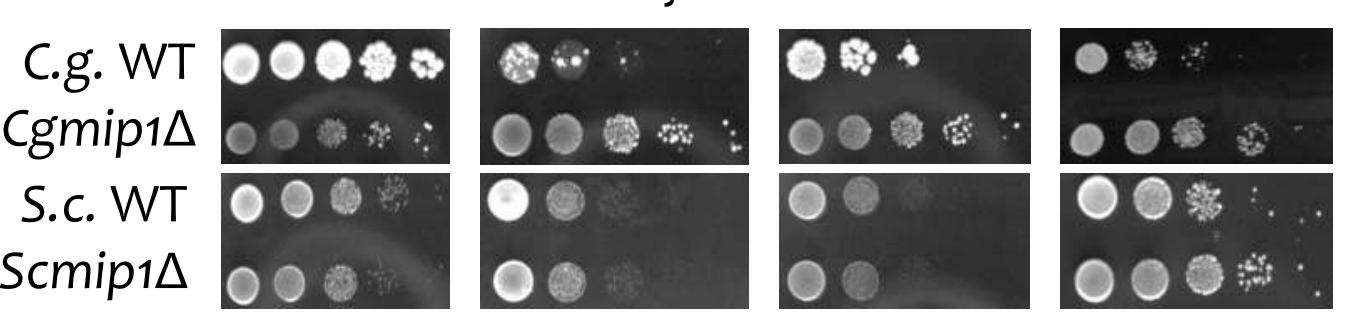

- mip1s strains show small colonies and loss of mitochondrial function (white colonies), typical of petite mutants phenotype.

- mip1 $\Delta$ strains show, as expected, decreased susceptibility to fluconazole. Contrary to Scmip1s, Cgmip1s shows steady growth on ER stress.

- The deletion of PDR1 triggers a growth defect under stress, whereas a PDR1 gain of function mutant (GOF) ${ }^{[1]}$ shows better growth .

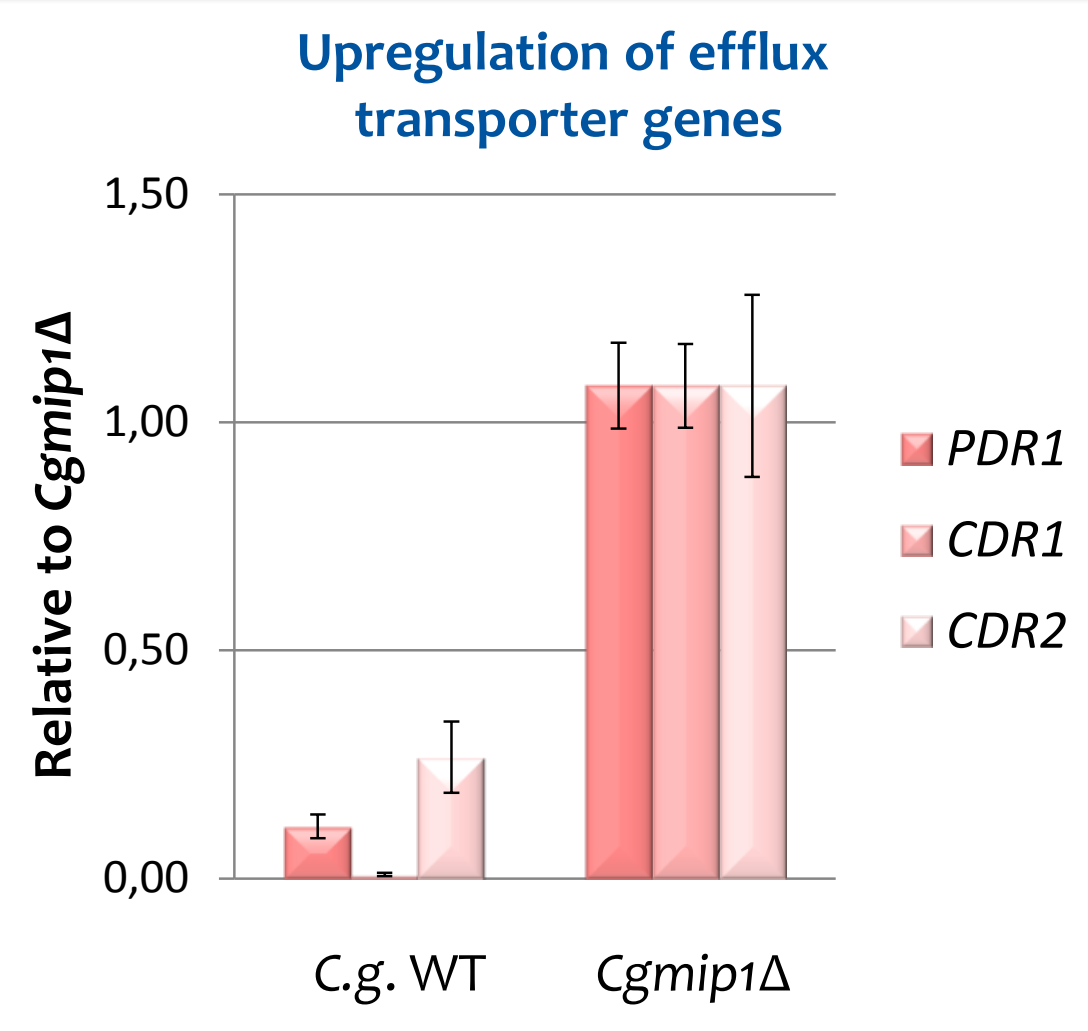

- Cgmip1A constitutively overexpresses $P D R 1$, and the efflux transporter genes CDR1 and CDR2.

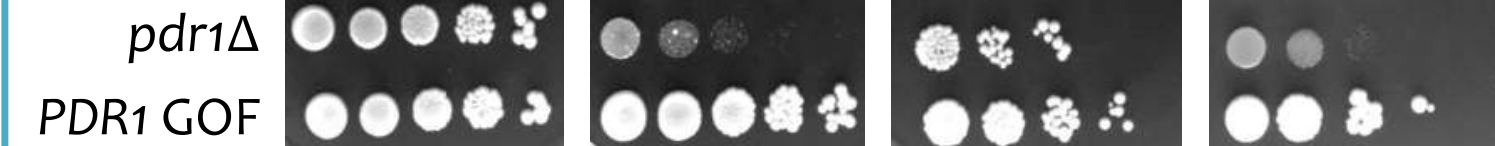
Transient petite phenotype as a survival strategy
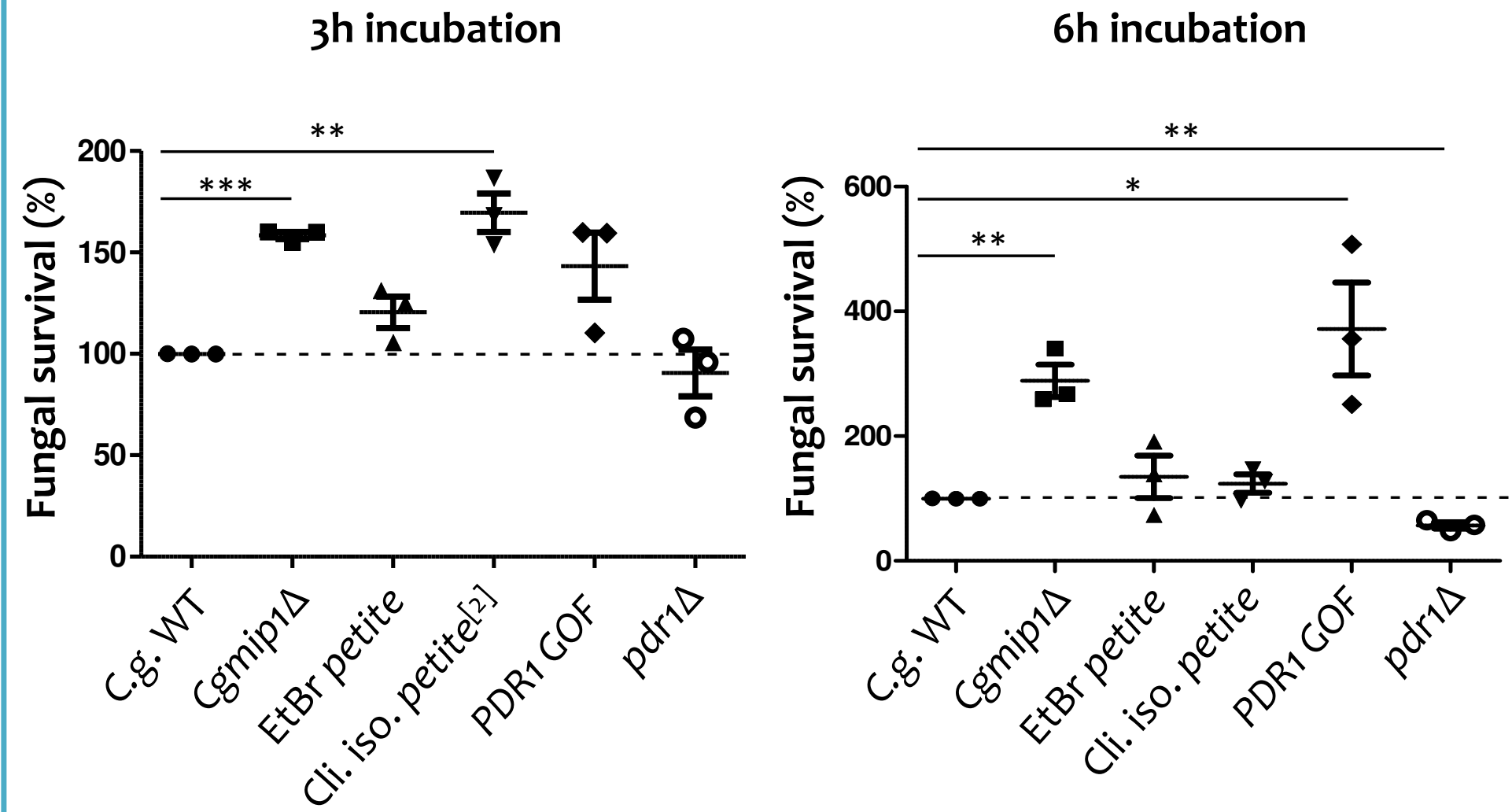

- Phagocytosis survival assay upon exposure to human monocyte-derived macrophages: Only Cgmip1 $\triangle$ and PDR1 GOF mutants show higher survival rate at all times, whereas lack of PDR1 leads to a lower survival rate.

\section{Doubling time}

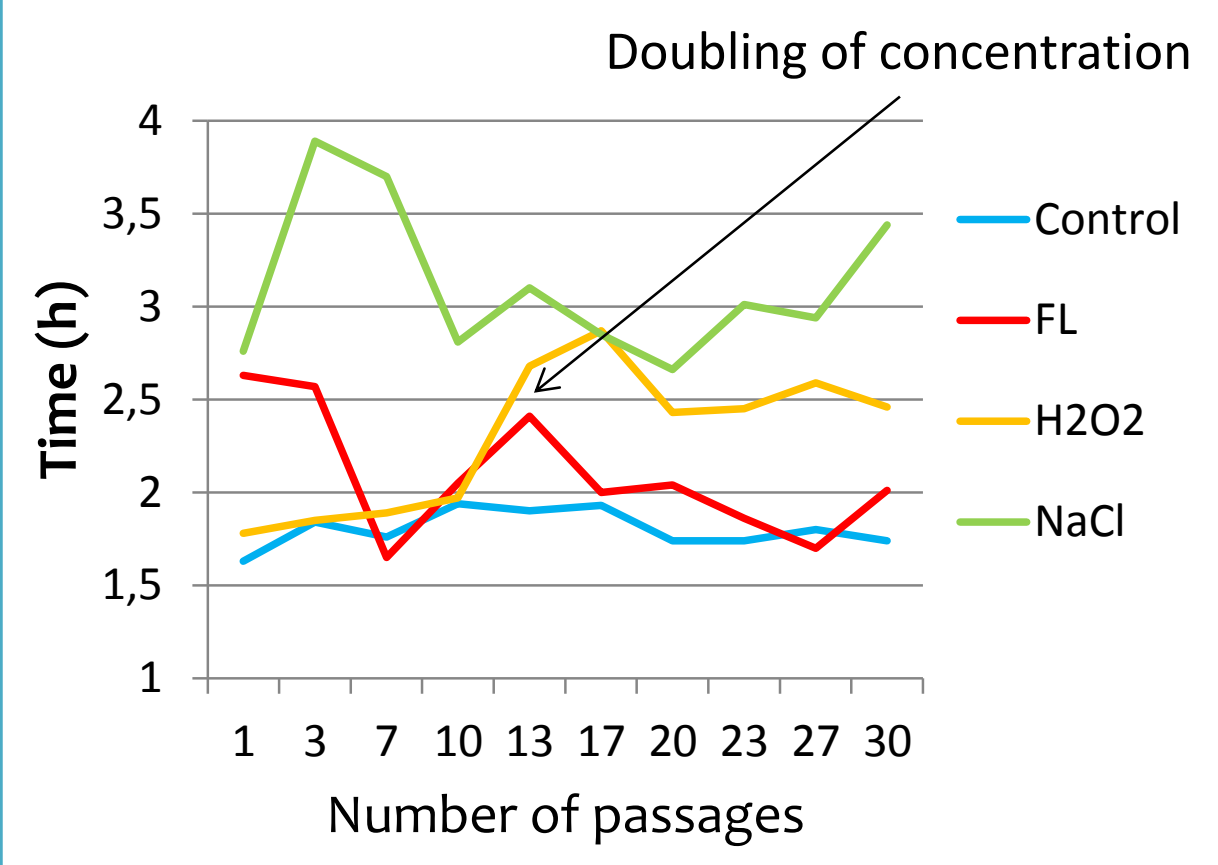

- Generation time of C. glabrata under different stresses: C. glabrata only adapts to FL and shows petite phenotype at the beginning of the longterm exposure experiment, which revert to respiratory metabolism.

\section{Conclusions}

* Cgmip1 $\Delta$ is a petite mutant that shows resistance to FL and ER stress likely due to the upregulation of the transcriptional factor Pdr1 and the efflux pumps Cdr1 and Cdr2. This seems also to result in a better fitness upon phagocytosis, which will be confirmed in further experiments.

- The transient petite phenotype seems to be involved in the adaptation of C. glabrata to high concentrations of FL by switching between respiratory and fermentative metabolism, possibly by an epigenetically regulated loss of function of CgMIP1. Epigenetic studies will be performed.

\section{References}

Ferrari S, et al. 2009. Plos Pathog Ferrari S, et al. 2011. Antimicrob. Agents Chemother.

\section{Contact}

Sofia.Lewin@hki-jena.de

\section{Acknowledgements}

This research was supported by the Graduate School of Excellence Jena School for Microbial Communication (JSMC) 